\title{
An Infrared Sensor for Eye Tracking in a Harsh Car Environment
}

\author{
K. RóżANOWSKI ${ }^{a, *}$ AND K. MurawsKI ${ }^{b}$ \\ ${ }^{a}$ Aviation Bioengineering Department, Military Institute of Aviation Medicine \\ Z. Krasińskiego 54, 01-755 Warsaw, Poland \\ ${ }^{b}$ Institute of Teleinformatics and Automatics, Military University of Technology \\ S. Kaliskiego 2, 00-908 Warsaw, Poland
}

\begin{abstract}
The paper presents the optical sensor which is built for EyeTracker operating in car harsh environment. The sensor is based on USB high speed video infrared camera. Additional hardware was designed for control and emission of infrared light as well. The designed hardware and software of the sensor are described in the paper. Some results of work, including video processing, are also presented.
\end{abstract}

PACS: 42.30.Sy, 42.30.Tz, 42.30.Va

\section{Introduction}

The object of research is an optical sensor designed for the subsystem of eye activity research (PBAO). The main task of PBAO is to support the assessment of the driver's mental and physical condition based on recording the activity of the eyes $[1,2]$. In determining the activity of the eyes with the following indicators there are tested the condition of the eyes closed/open, the duration of when the eyes are closed, the frequency of closing the eyelids, and the PERCLOS factor (percentage of eye closure). The most effective methods of researching the eye motion activity include the electrooculography method, which consists of measuring changes of electric field charges induced by movements of the eye $[1,3]$, tracking the movement of the eyes by a single camera or a set installed on a headband.

These methods are reliable in operation, but they have no chance to be put into use on a massive scale due to the troublesome need to install sensors on the skin or head of the driver. They limit the freedom of movement and reduce the concentration of people using the system during a particular task. These methods are only capable to be used during research. The authors abandoned the development of the above methods focusing on the construction of the handsfree PBAO subsystem, whose integral part is the sensor discussed in Sect. 3 of the article. The use of handsfree systems to track the movements of the eyes is becoming more common. Unfortunately, the solutions currently available are characterized with high sensitivity to the changes in light levels. For this reason they must be used in laboratory or simulator conditions.

* corresponding author; e-mail: krozan@wiml.waw.pl
They are actively used in the research: marketing [4], designing of websites [5], graphical user interfaces [6], etc. To identify the position of the eyes in the recorded video footage and determine their status usually dedicated image processing algorithms [7] are used. The characteristic phenomenon for the eyes are used in this case: bright pupil - Fig. 1a and/or dark pupil — Fig. 1b. The bright pupil effect occurs when a lens optical axis coincides or is close to the optical axis of an infrared radiator, Fig. 1a. In such a configuration the reflection from the bottom of the eye is observed. The reflection is recorded by a sensor in the form of a bright spot. The dark pupil effect works similarly. If the optical axes of the camera and the illumination do not coincide or are insufficiently close to each other, Fig. 1b, the eye acts like a black body model.

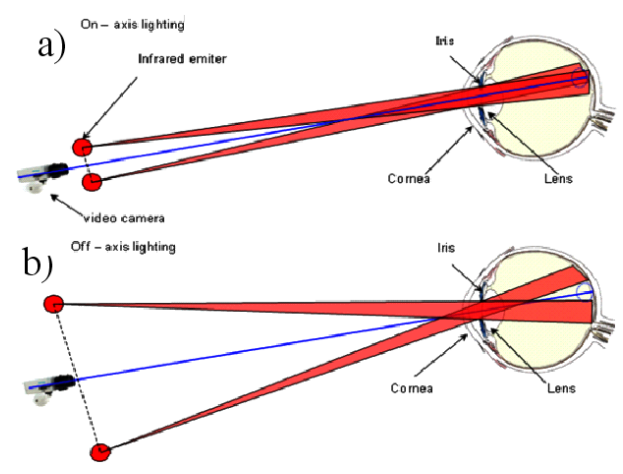

Fig. 1. The principle of the formation of the bright (a) and dark pupil (b).

Due to this the light is internally reflected and escapes through the pupil. Its image is recorded by the sensor in the form of a spot of low brightness, and then ana- 
lyzed. Other imaging methods used for determining the position of the face, including the eyes, can be found in the literature. Most of them focus on finding characteristics defined during the learning process. The most popular are: support vector machine [8], Viola-Jones [9], active contour [10], active apperance models [11], eigenfaces [12].

\section{Optical sensor, preliminary research}

In the constructed PBAO the possibility of using the light and dark pupil effect is taken into account. Ultimately, the choice was made on the basis of pilot studies. During the experiments, the IR source $(\lambda=850 \mathrm{~nm})$ was positioned relative to the camera in accordance with Fig. 1. For each position of the emitter a series of images were recorded, which were processed by the developed methods of digital image processing, Fig. 2.
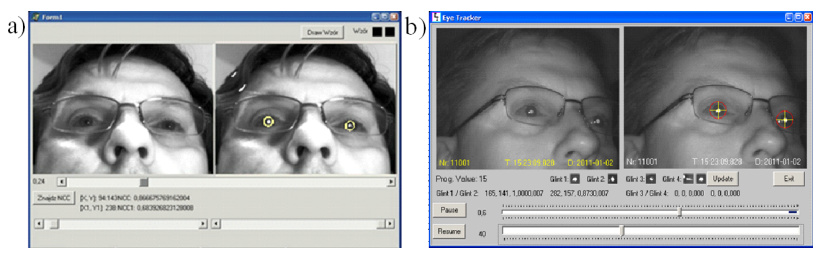

Fig. 2. Input image - the phenomenon of the dark pupil (left), the result of processing (right) (a), input image - the phenomenon of the bright pupil (left), the result of processing (right) (b).

The study used an Optitrack V120:SLIM monochrome camera. The camera is mounted with a high-pass filter (blocking visible light) and a lens adapted to work in infrared with a focal length $f=16 \mathrm{~mm}$ and brightness $F=2.0$. During the experiments, the camera worked in the following settings: resolution $-320 \times 240$ pix, frame rate $-30 \mathrm{fps}$ (frames per second), exposure - 120 .

As a temporary light source two infrared emitters at $\lambda=850 \mathrm{~nm}$ were used. Illuminators were supplied with a constant amplitude voltage $U_{Z}=+12 \mathrm{~V}$, Fig. 3 . Voltage passed through terminals $\mathrm{A}(+12 \mathrm{~V})$ and $\mathrm{B}$ (GND). The first illuminator was built using twenty-four IR LEDs. It was set up in three branches of eight LEDs each.

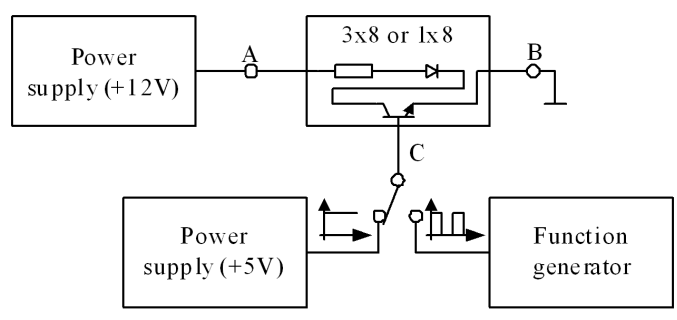

Fig. 3. Infrared emitter research system.

The second had two branches, each with six IR LEDs connected in a series, Fig. 4. The light source was activated with input $\mathrm{C}$, accompanied by a control signal.
During image analysis, performed at a distance of one meter from the object, no significant changes were observed resulting from the different number of LEDs. For this reason, it was decided to use the emitter with fewer LED diodes. The solution helped to minimize the radiation power emitting to the driver's eyes and to maintain the required compliance [13].

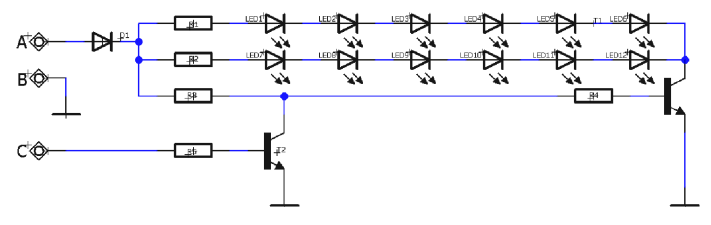

Fig. 4. Temporary infrared emitter system.

At the time of the study the impact of the shape and frequency of the signal supplied to input $\mathrm{C}$ on the brightness of the image collected in the pupil was also identified.

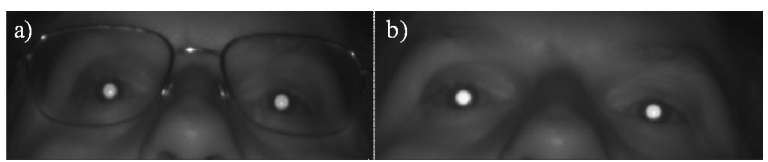

Fig. 5. Images obtained with continuous illumination control of the glasses (a), without glasses (b).

The reference point was the measurement made with controlled DC voltage with an amplitude of $U_{\mathrm{C}}=+5 \mathrm{~V}$. The practical effect of the operation of the new radiator design was also verified for cases with people wearing contact lenses. The first images were taken with glasses, Fig. 5a. Under the same conditions, the experiment was repeated without glasses, Fig. 5b. Comparing images $5 \mathrm{a}$ and $5 \mathrm{~b}$ we can see a clear impact of the glasses on the resulting image of the pupils — Table I.

Average levels of pupil brightness (0 - black, 255 - white).

\begin{tabular}{l|c|c|c|c}
\hline \hline \multirow{2}{*}{ Control type } & \multicolumn{2}{|c|}{ With glasses } & \multicolumn{2}{c}{ Without glasses } \\
\cline { 2 - 5 } & left eye & right eye & left eye & right eye \\
\hline continuous light & 134.84 & 125.69 & 154.83 & 174.94 \\
pulse light & 197.03 & 209.89 & 235.49 & 236.91 \\
value of change & $46.12 \%$ & $66.99 \%$ & $52.10 \%$ & $35.42 \%$
\end{tabular}

In the first case (with glasses), the average brightness level of the pupils was 130.26 . After removing the glasses, this level increased to 164.88 giving it nearly a $27 \%$ increase in brightness. In addition, in the first and second case, the initial brightness of pupils decreased. The reason for this phenomenon was the change in diameter of the pupils under the influence of the infrared light.

The test was repeated by changing the method of control. A square-wave signal was fed to the $\mathrm{C}$ input with filling $1 / 2$, an amplitude of $U_{\mathrm{C}}=+5 \mathrm{~V}$ and a frequency 


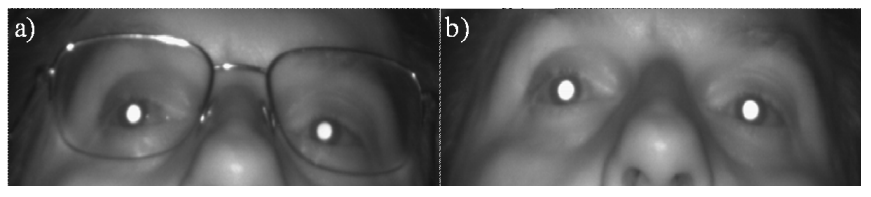

Fig. 6. The images obtained using impulse illumination control in glasses (a), without glasses (b).

range from $f=1 \mathrm{kHz}$ to $f=100 \mathrm{kHz}$. The results obtained for $f=50 \mathrm{kHz}$ is presented in Fig. 6 .

During IR radiator impulse control higher average brightness levels of the pupils were obtained. The study received 203.46 in glasses, and after they were taken off this value increased to the value of 236.20 . As in the previous case an increase was noted in the brightness level of $16.1 \%$.

In summary, the average increase in the brightness of the pupil resulting from the application of impulse control was: with glasses - 56.6\%; without glasses - $43.8 \%$, giving an average increase of more than $50 \%$ in brightness compared to the illumination controlled by a DC voltage of the same amplitude.
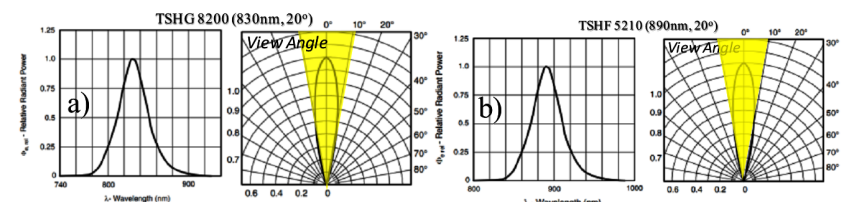

HIRB5-43G-D $\left(850,50^{\circ}\right)$
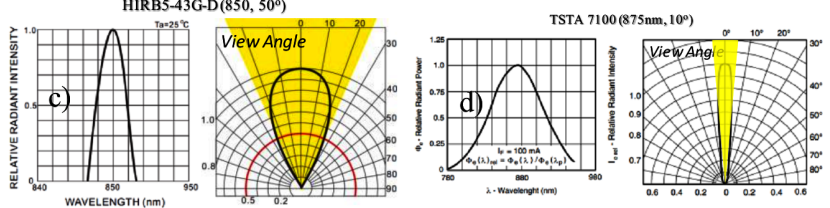

HIRB5-4

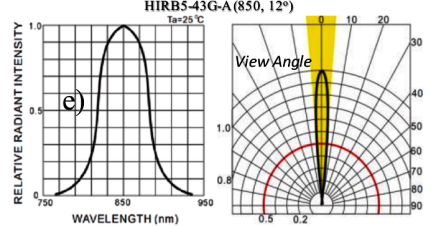

Fig. 7. Optical diode characteristics: TSTA 7100 (a), TSHG 8200 (b), TSHF 5210 (c), HIRB5-43g-D (d), HIRB5-43g-A (e).

During the preliminary research work was carried out related to identifying optimal elements for infrared emission to develop a new radiator design. For this reason optical properties of a selected group of LEDs were analyzed. From experience the following elements were selected: TSHG $8200(830 \mathrm{~nm})$; TSHF $5210(890 \mathrm{~nm})$; HIRB5-43g-A $(850 \mathrm{~nm})$; HIRB5-43g-D $(850 \mathrm{~nm})$, TSTA $7100(875 \mathrm{~nm})-$ Fig. 7. From the standpoint of sensor design most important were the emitted wavelengths, luminous intensity and the angle at half the optical power. Also important were the forward voltage $U_{\mathrm{F}}$ and the forward current $I_{\mathrm{F}}$.
The values of these parameters are summarized in Table II. Then, they were tested again. As a result, for the construction of the final version of the radiator the HIRB5-43g-A diode was selected.

Select parameters of the analyzed IR diodes.

TABLE II

\begin{tabular}{c|c|c|c|c|c|c}
\hline \hline Diode & $\lambda$ & $U_{\mathrm{F}}$ & $I_{\mathrm{F}}$ & $U_{\mathrm{R}}$ & $\begin{array}{c}\text { Radiant } \\
\text { intensity } \\
{[\mathrm{mW} / \mathrm{sr}]}\end{array}$ & $\begin{array}{c}\text { Angle } \\
\text { of half } \\
\text { intensity } \\
{[\mathrm{deg}]}\end{array}$ \\
\hline HIRB5-43G-A & 850 & 1.5 & 50 & 4 & 140 & 12 \\
HIRB5-43G-D & 850 & 1.5 & 50 & 4 & 55 & 50 \\
TSHF 5210 & 890 & 1.4 & 100 & 5 & 180 & 20 \\
TSTA 7100 & 875 & 1.4 & 100 & 5 & 50 & 10 \\
TSHG 8200 & 830 & 1.5 & 100 & 5 & 180 & 20
\end{tabular}

For each diode an illuminator was constructed according to the scheme shown in Fig. 8.

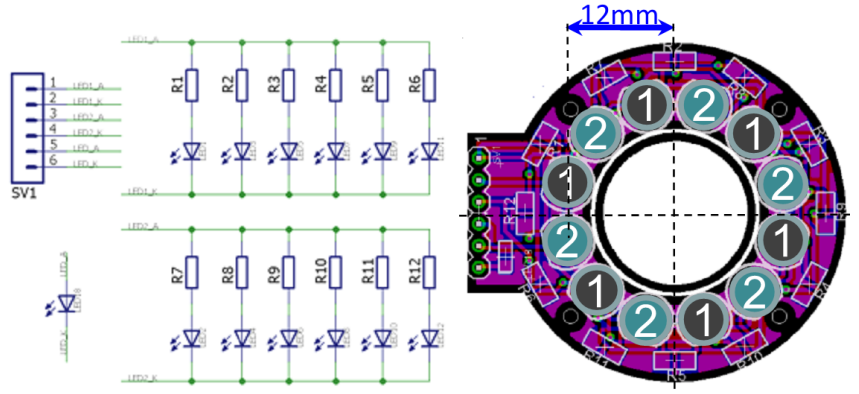

Fig. 8. Diagram and arrangement of infrared radiator diodes on the circuit board.

\section{Infrared sensor configuration}

The current sensor for testing eye activity consists of a fast monochrome camera, infrared radiator, radiator controller, lens and filter. Relative position of the elements is shown in Fig. 9.

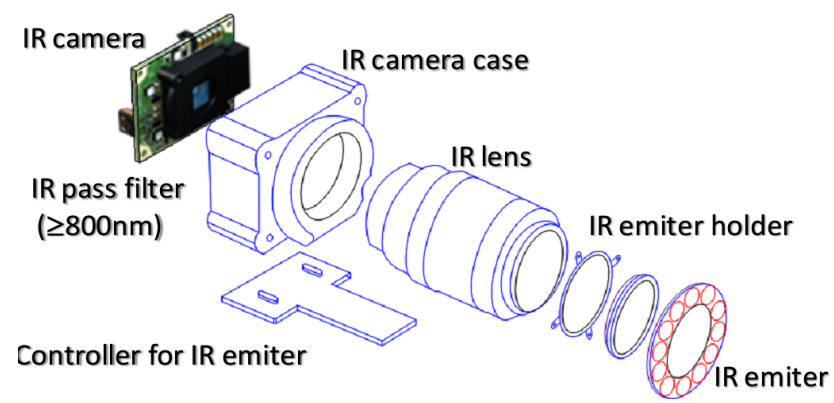

Fig. 9. Relative position of the sensor elements constructed to test the activity of the eyes (Camera V120:SLIM).

The basic element is the video camera sensor. The project uses an OptiTrack USB V120:SLIM camera with 
the following parameters: width $42 \mathrm{~mm}$, height $32 \mathrm{~mm}$, weight $5 \mathrm{~g}$, resolution [pix] $160 \times 120,320 \times 240,640 \times$ 480 , frame rate [fps] $30,60,120$, IR pass filter included, shutter type global, default shutter speed $1 \mathrm{~ms}(20 \mu \mathrm{s}$ minimum).

The HF16HA-1B 1:1.4/16 mm lens is included with the camera with a screwed IR emitter handle to which the designed illuminator is attached to. The diagram of radiator and the distribution of LEDs on the PCB board was presented in Fig. 8. Numbers 1 and 2 in Fig. 9 relate to the first and second set of LEDs. The operation of the radiator is managed by a control system equipped with a microcontroller, Fig. 10. The project uses an AT89S8253-24AU microcontroller in a TQFP44 case, clocked at $f=$ $22.1184 \mathrm{MHz}$. The adopted control was conventionally called the pulse control with keying pulse (in short pulse pulse control).

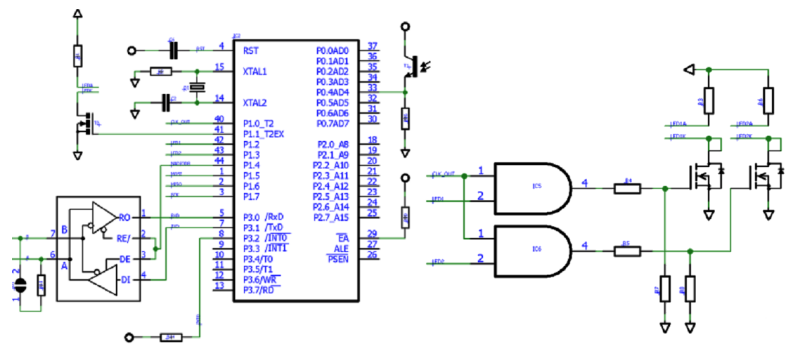

Fig. 10. Diagram of a custom IR illuminator control system.

Square wave is generated by the microcontroller at output P1.0 (pin 40). Using the T2 microcontroller timer, for the adopted $f$, it is possible to generate a square wave with a frequency of up to about $920 \mathrm{kHz}$. Its value is calculated from the following formula:

$$
\left.F_{\mathrm{CLK}}=\frac{f}{4 \times[65536-(\mathrm{RCAP} 2 \mathrm{H}, \mathrm{RCAP} 2 \mathrm{~L})}\right] .
$$

Exemplary values of RCAP2 registry values and corresponding frequencies are included in Table III.

TABLE III

Exemplary registry values and corresponding frequencies.

\begin{tabular}{c|c|c|c|c|c}
\hline \hline$f[\mathrm{~Hz}]$ & Value & $f[\mathrm{~Hz}]$ & Value & $f[\mathrm{~Hz}]$ & Value \\
\hline 20000 & FEEB & 39000 & FF72 & 58000 & FFA0 \\
21000 & FEF8 & 40000 & FF75 & 59000 & FFA2 \\
22000 & FF04 & 41000 & FF79 & 61000 & FFA5 \\
25000 & FF22 & 44000 & FF82 & 65000 & FFAA \\
26000 & FF2B & 45000 & FF85 & 66000 & FFAC \\
27000 & FF33 & 46000 & FF87 & 68000 & FFAE \\
28000 & FF3A & 47000 & FF8A & 70000 & FFB1
\end{tabular}

The oscillogram of the generated square wave is presented in Fig. 11a. The generated carrier wave, is lead to gate AND. The second input gate is connected to pins $\mathrm{P} 1.2$ and $\mathrm{P} 1.3$ of the microcontroller. At these ends a square wave was of $f=3.4 \mathrm{kHz}$ and with an adjustable duty cycle. The product of the included signals was controlled by unipolar transistor gates, which keyed the IR LED current, Fig. 11b.
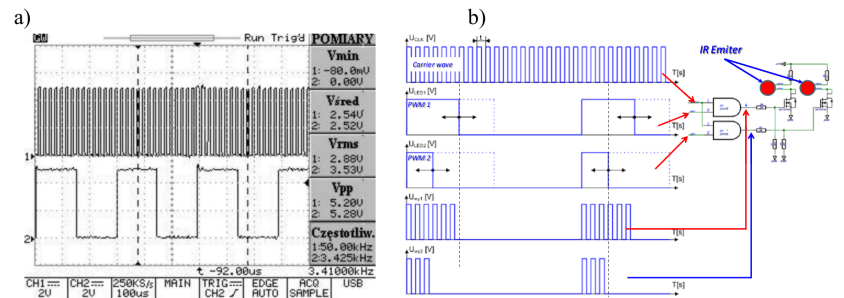

Fig. 11. (a) The course of the generated square wave with P1.0 on the output at registry values RCAP2 $=$ 0xFF4D (channel 1), the generated keying wave (channel 2); (b) the idea of controlling the illuminator brightness.

Examples of oscillograms obtained at different pulse duty factors are presented in Fig. 12. Figure 12 shows that when the keying pulse extends more, carrier waves can turn on/off the diode. As a result, the emitter generates more energy, and the picture becomes more illuminated. All illumination settings are remotely set via the RS-485 interface. Int: $10 \%, \Delta t=38.40 \mu$ s, Int: $40 \%$; $\Delta t=122.0 \mu \mathrm{s}$.

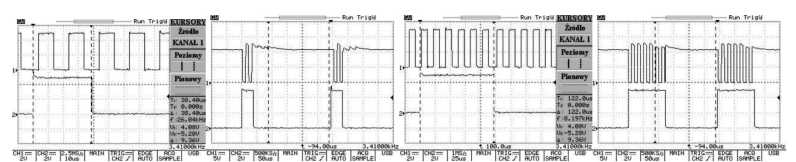

Fig. 12. Illuminator power control: shape of the keying pulse wave - channel 2, left; the result of the controlling LED diodes - channel 1 , right.

During the measurements, Fig. 12, the oscilloscope probes were connected as follows:

\begin{tabular}{l|c|c} 
& Left side (SL) & Right side (SP) \\
\hline channel 1 & P1.0 (CLK) & unipolar transistor drain \\
channel 2 & P1.2 (KEY) & P1.2 (KEY)
\end{tabular}

\section{Experimental details and results}

The integrity of the developed sensor was tested in real and laboratory conditions. When you attach it to the produced central unit, the laboratory tests were performed. Minimal impact of infrared was provided coming from the sun through proper room blackout during the experiments. The room was equipped with cold white artificial lighting. The research included 20 randomly selected individuals of different sex and age (from 18 to 25 years of age). The results showed that in such conditions the prepared sensor worked properly. During the experiments in the laboratory the location and the status of the eyes were correctly determined. There were a few 


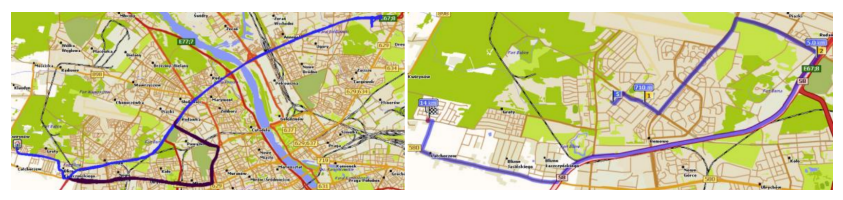

Fig. 13. Day route - distance approximately $50 \mathrm{~km}$, route S8, Latchorzew - Marki (left); night route distance approximately $14 \mathrm{~km}$, route S8, MUT Latchorzew (right).
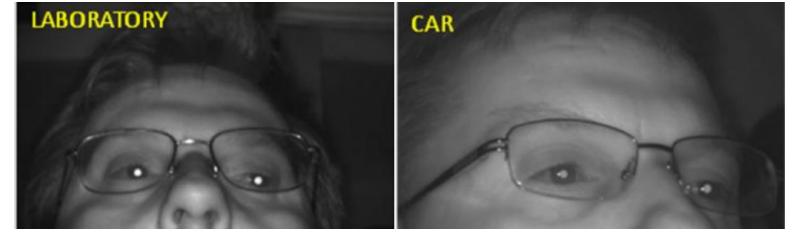

Fig. 14. View of the pupils in laboratory (left) and road tests (right).

cases of partial (determining the position of only one eye) or a lack of identification of the position of the eyes resulting from the imperfections of the software for image analysis. Then the sensor was mounted in the passenger car (Fig. 15b). Verification of the system's operation while driving on the road was done on the streets of Warsaw in normal traffic. Tests were carried out during the day and night on the routes shown in Fig. 13. Road tests, especially during the day, helped to verify the design assumptions. There was a noticeable decrease in brightness of the pupils (Fig. 14), which made it difficult, but still allowed for efficient image scanning to determine the position of the eyes and to determine their status. The software cooperating with the sensor was corrected during the next stage of optimization after the laboratory tests. They were modified so that the eye position was scanned with a predetermined probability threshold. By default, this threshold was set at 0.7. This means that the image section shall be considered an eye if the image similarity coefficient calculated between the image and the pattern is more or equal 0.7 .

This makes it possible to find position of the eyes, even after a long break, i.e., loss of information about their position. These breaks occurred mainly in conditions of intense sunlight or blurring the image by glasses. Similar behavior of the system was detected using contact lenses. In the first case, the pupil was too small to be identified in the image. In the second case there were difficulties with determining a clear boundary between the pupil and the surrounding area. In other situations, positions of the pupils and the state of the eyes were determined correctly.

\section{Conclusions}

This paper presents an optical sensor developed for subsystem of eye activity research. The task of the sensor, along with dedicated software is to track the activity of the eyes and the determination of the psycho-physical condition characteristics of the driver. To achieve the determined target the infrared radiator controller, the radiator and the sensor itself was developed and tested. This was followed by tests of the produced sensor in the laboratory and road conditions (day and night). The obtained results are shown in Fig. 15 and Fig. 16.

The circles in the pictures identify the position of the eyes by the software. Each picture bears a time marker. The marker includes: frame number, time and the date of the test. At the top of the picture the eye activity history is drawn in real-time covering the last 200 measurements. A typical video recording at a speed of 25 fps gives us $8 \mathrm{~s}$ of recording.

Performed experiments have shown that the constructed sensor works as required. In special circumstances, in the absence of interference, it is possible to observe the activity rate of the eyes of up to $60 \mathrm{fps}$. Theoretically, it is also possible to observe the activity rate of the eyes at $120 \mathrm{fps}$, but this mode requires stronger eye exposure light. This is the reason why it was abandoned.

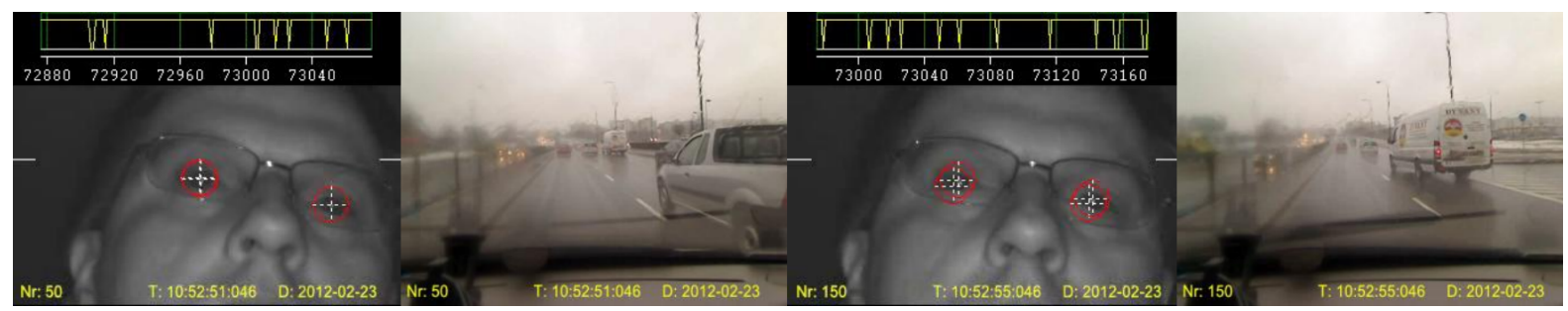

Fig. 15. Sensor operation during the day and view from the car. 


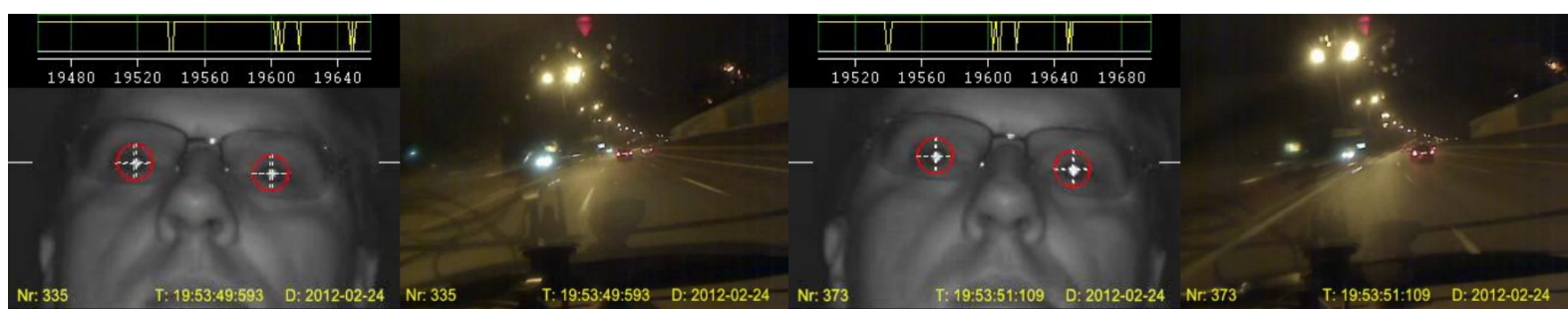

Fig. 16. Example of the sensor operation after dusk and view from the car.

\section{Acknowledgments}

The project was co-financed by the European Union through the European Regional Development Fund under the Innovative Economy Program. Development projects contract no. POIG.01.03.01-10-085/09.

\section{References}

[1] K. Murawski, Przeglad Elektrotechniczny 9, 184 (2010) (in Polish).

[2] S. Zhao, R.R. Grigat, Machine Learn. Data Min. Pattern Recogn. 3587, 633 (2005).

[3] http://www.bem.fi/book/28/28.htm, (2012).

[4] http://www.humangraph.pl/shopper-track, 39.html\#, (2012).

[5] http://freeflow.pl/badania-uzytecznosci/ eyetracking/, (2012) (in Polish).

[6] http://www mechanikaumyslu.pl/tag/eye-tracking/, (2012).
[7] K. Mikołajczyk, T. Tuytelaars, C. Schmid, A. Zisserman, J. Matas, F. Schaffalitzky, T. Kadir, L. Van Gool, Int. J. Comput. Vision 65, 43 (2005).

[8] P. Campadelli, R. Lanzarotti, G. Lipori, IOS Press 18, 234 (2007).

[9] P. Viola, M. Jones, in: Proc. of IEEE Workshop on Statistical and Computation Theories of Vision, IEEE, Vancouver 2001.

[10] http://research.microsoft.com/en-us/um/ people/ablake/contours/, (2011).

[11] S. Hommel, U. Handmann, 12th International Symposium on, Computat. Intellig. Inform., IEEE, 2011, p. 189 .

[12] P. Belhumeur, J. Hespanha, D. Kriegman, IEEE Trans. Pattern Anal. Machine Intellig. 19, 711 (1997).

[13] Dz.U. 2002 nr 217 poz. 1833 (in Polish). 